\title{
Glial Scar Formation Occurs in the Human Brain after Ischemic Stroke
}

\author{
Lijie Huang1,2\#, Zhe-Bao Wu ${ }^{1,2 \#}$, Qichuan ZhuGe ${ }^{1,2 \varpi}$, WeiMing Zheng ${ }^{1,2}$, Bei Shao ${ }^{2}$, Brian Wang ${ }^{3}$, Fen Sun ${ }^{3}$, \\ Kunlin Jin $1,2,3 \times$ \\ 1. Department of Neurosurgery, First Affiliated Hospital, Wenzhou Medical University, Wenzhou 325000, China \\ 2. Zhejiang Provincial Key Laboratory of Aging and Neurological Disorder Research, First Affiliated Hospital, Wenzhou Medical Univer- \\ sity, Wenzhou 325000, China \\ 3. Department of Pharmacology and Neuroscience, University of North Texas Health Science Center at Fort Worth, Texas 76107, USA
}

\# These authors made equal contributions to this paper

$\triangle$ Corresponding author: Qichuan ZhuGe, M.D., Professor, Department of Neurosurgery, First Affiliated Hospital, Wenzhou Medical University, Wenzhou, China, Tel: 86-577-88069618; Fax: 86-577-88069617; Email: zhugeqichuan@vip.163.com or Kunlin Jin, M.D., Ph.D., Professor, Department of Pharmacology \& Neuroscience, University of North Texas Health Science Center, 3500 Camp Bowie Boulevard, Fort Worth, TX 76107, USA, Tel: 817-7352579, Fax: 817-7350408, Email: kunlin.jin@unthsc.edu

(c) Ivyspring International Publisher. This is an open-access article distributed under the terms of the Creative Commons License (http://creativecommons.org/ licenses/by-nc-nd/3.0/). Reproduction is permitted for personal, noncommercial use, provided that the article is in whole, unmodified, and properly cited.

Received: 2013.1I.16; Accepted: 2013.12.23; Published: 2014.02.II

\begin{abstract}
Reactive gliosis and glial scar formation have been evidenced in the animal model of ischemic stroke, but not in human ischemic brain. Here, we have found that GFAP, EDI and chondroitin sulphate proteoglycans (CSPG) expression were significantly increased in the cortical peri-infarct regions after ischemic stroke, compared with adjacent normal tissues and control subjects. Double immunolabeling showed that GFAP-positive reactive astrocytes in the peri-infarct region expressed CSPG, but showed no overlap with EDI-positive activated microglia. Our findings suggest that reactive gliosis and glial scar formation as seen in animal models of stroke are reflective of what occurs in the human brain after an ischemic injury.
\end{abstract}

Key words: glial scar, reactive gliosis, ischemic stroke, human, patients

\section{INTRODUCTION}

Brain injuries trigger an extensive glial response referred to as reactive gliosis, which is characterized by an increased expression of specific markers, such as glial fibrillary acidic protein (GFAP) for astrocytes, various extracellular matrix molecules (ECM) like chondroitin sulphate proteoglycans (CSPG) and microglia. In severe cases, reactive gliosis eventually leads to the formation of a glial scar surrounding the injury site. The glial response to ischemic stroke has been widely studied in a number of animal models using photothrombosis, and variations of focal transient or permanent middle cerebral artery occlusion (MCAO) [1-3]. Reactive astrogliosis, however, is a ubiquitous but poorly understood hallmark of all central nervous system (CNS) pathologies.
Recent studies show that reactive gliosis exert both beneficial and detrimental effects on restoring brain functions after injury. In the acute phase, glial scar formation is crucial for sealing the lesion site to remodel the tissue, and temporally and spatially controlling the local immune response. The glial barrier seals off the area of damage to prevent further microbial infections and spread of cellular damage, maintain extracellular ion and fluid balance, prevent an overwhelming inflammatory and growth factor responses, as well as free radical scavenging [4]. Furthermore, the glial scar stimulates revascularization of blood capillaries to increase the nutritional, trophic, and metabolic support of the nervous tissue. On the other hand, the glial scar is also acts as an impediment 
to axon regeneration and thus prevents the recovery of CNS function in the chronic phase, because the reactive astrocytes secrete several growth-inhibitory molecules that chemically prevent axonal extensions. Moreover, the basal membrane component generates an additional physical barrier to regenerating axons that disallows them to cross the lesion. However, these findings are based mainly on rodent brains. Little is known about how the reactive gliosis respond to acute ischemic stroke in the human brain. In this present study, we investigated the expression profiles of glial scar components in the human ischemic brain, and found that reactive gliosis and glial scar formation in the human brain after ischemic injury retain similar properties to those animal models of stroke.

\section{MATERIALS AND METHODS}

\section{Human brain specimens}

Normal human brain specimens $(n=6)$, who were 25-48 years old without neurological diseases, were obtained from the Brain and Tissue Bank for Developmental Disorders at the University of Maryland at Baltimore (Baltimore), USA. Ischemic brain specimens $(n=6)$ were obtained from ischemic stroke patients of 34-74 years old undertaking biopsy for diagnostic purposes. More details, such as age, sex, duration of symptoms and infarct sites, have been documented in our previous study [5]. Research was conducted in compliance with the policies and principles contained in the Federal Policy for the Protection of Human Subjects. All the brain specimens were obtained with informed consent and this study was approved by the Institutional Research Review Board at University of North Texas Health Science Center at Fort Worth, Texas, USA.

\section{Immunohistochemistry}

Human brain specimens were embedded in paraffin and cut in $6-\mu \mathrm{m}$ sections. Immunohistochemistry was performed on these sections as previously described [5]. The primary antibodies used were 1) mouse monoclonal anti-CSPG (Millipore; 1:100), 2) rabbit polyclonal anti-GFAP (Sigma; 1:1,000), and 3) mouse monoclonal anti-ED1 (Serotec; 1:200). Sections were imaged using a Nikon E300 epifluorescence microscope. Controls included omitting incubation with primary or secondary antibodies.

\section{Double-label immunohistochemistry}

Double-label immunohistochemistry was performed on brain sections as previously described [5]. The primary antibodies used were listed above. The secondary antibodies used were Alexa Fluor 488-, 594-, or 647-conjugated donkey anti-mouse or anti-rabbit IgG (Molecular Probes, 1:200-500). Slides were mounted using proLong Gold anti-fade reagent with DAPI (Molecular Probes). Fluorescence signals were detected using an LSM 510 NLO Confocal Scanning System mounted on an Axiovert 200 inverted microscope (Carl Zeiss Ltd) with a 2-photon Chameleon laser (Coherent Inc.). Images were acquired through LSM 510 Imaging Software (Carl Zeiss Ltd). Controls included omitting incubation with primary or secondary antibodies.

\section{RESULTS}

To investigate whether glial scar was formed in the ischemic brain in humans, we first determined the expression pattern of CSPG, a major constituent of the glial scar [4]. As shown in Figure 1A, CSPG in brains of 6 patients with stroke was significantly expressed in the cortical peri-infarct regions (penumbra) and infarct core of human ischemic brains, compared with adjacent normal tissue.

Next, we performed immunocytochemistry to study the profile of reactive astrocytes after ischemic stroke. As shown in Figure 1B, a significant increase in GFAP-positive reactive astrocytes was observed in the cortical penumbra regions, compared with adjacent tissues. Most reactive astrocytes in the regions displayed hypertrophy of the cell body and processes, which formed an encapsulation layer around the lesion site. The extensive overlap and interdigitations of processes of these reactive astrocytes were found in the cortical peri-infarct regions in human ischemic brains. In normal tissue, the extensive network of finely branched processes of individual astrocytes was observed with no overlap.

Microgliosis, a process that sees the migration of macrophages and local microglia to the injury site in response to ischemic injury, is a form of gliosis. Hence, ED1, a cellular marker specific for activated microglia and macrophages, was used for immunostaining. As shown in Figure 1C, ED1-positive activated microglia were also found in the penumbra and infarct core in brain specimens of stroke patients.

Double immunostaining shows that GFAP and CSPG proteins were overlapped in the intermediate penumbra area (Figure 2A and 2B), forming the border zone between the infarct core and adjacent normal tissue, which is consistent with the expression and distribution of CSPG and GFAP in rodents after ischemic injury. ED1-positive activated microglia were also distributed in the glial scar region (Figure 2C). At a higher magnification, GFAP-immunopositive reactive astrocytes displayed morphological features of reactive gliosis, including present hypertrophic somata and extended processes to the adjacent infarct core (Figure 2D). 

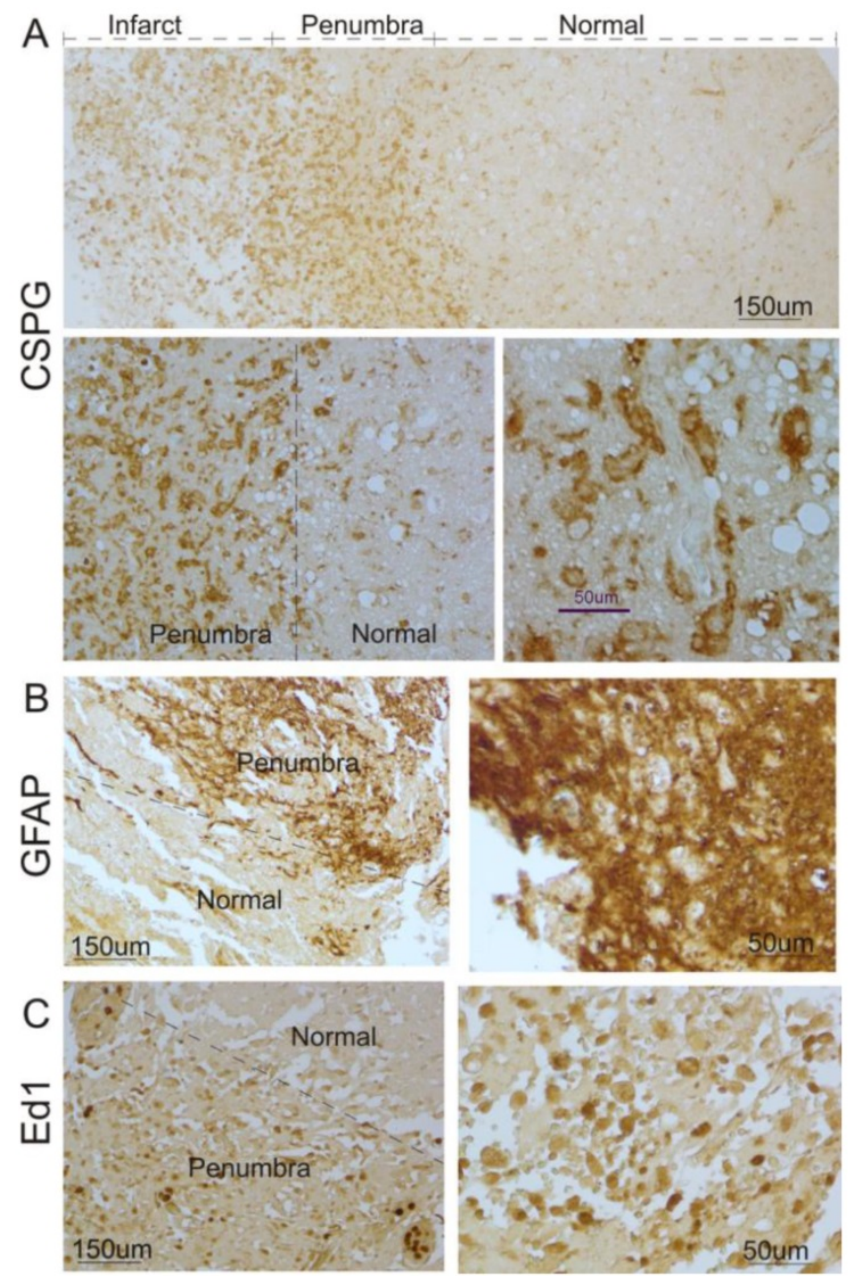

Figure I. Glial scar formation in the peri-infarct region of the human brain after ischemic stroke. A. Human ischemic brain sections were stained with anti-CSPG. Representative images show that increased CSPG expression was predominantly found in the peri-infarct region and some were within the infarct area. Top panel: low magnification; Bottom panel: high magnification. B. Immunocytochemistry was performed using anti-GFAP. Representative images indicate that GFAP-positive reactive astrocytes significantly increased in the peri-infarct region after ischemic stroke in humans, compared with the normal region. Left panel: low magnification; Right panel: higher magnification. C. Immunocytochemistry was performed using anti-EDI. Representative images indicate that EDI-positive activated microglia were significantly increased in the peri-infarct region after ischemic stroke in humans, compared with the normal region. Left panel: low magnification; right panel: high magnification.

\section{DISCUSSION}

The glial scar consists predominately of reactive astrocytes, microglia and extracellular matrix molecules, especially CSPGs. We found in this study that GFAP-positive reactive astrocytes and ED1-positive activated microglia were significantly increased in the cortical peri-infarct regions of human ischemic brain, compared to adjacent normal tissues and control subjects. Double immunolabeling showed that GFAP-positive reactive astrocytes in the peri-lesional region expressed CSPG, but did not overlap with
ED1-positive activated microglia. GFAP- and ED1-positive cells, along with CSPG, formed a thin layer around the ischemic lesion, suggesting the presence of a glial scar in the cortex of human brains after ischemic stroke.
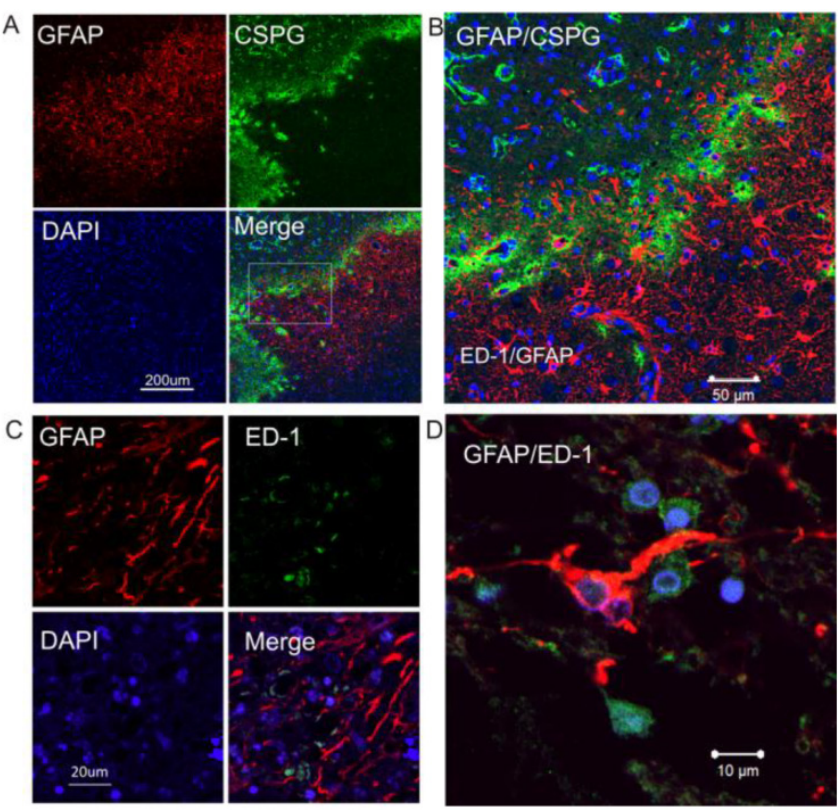

Figure 2. Increased expression of CSPG in reactive astrocytes in the peri-infarct region of human ischemic brain. A. Double immunocytochemistry was performed on the ischemic brain sections using anti-GFAP (red) and anti-CSPG (green). The images were recorded using a 2-photon confocal microscope. Representative images show that CSPG was predominantly expressed in GFAP-positive reactive astrocytes. B. Higher magnification view of insert in $2 \mathrm{~A}$. C. Confocal images of double-label immunohistochemistry on the ischemic brain section using anti-GFAP (red) and anti-EDI (green). D. Higher magnification shows GFAP-positive reactive astrocytes (red) not co-localizing with EDI-postive activated micrglia (green). DAPI (blue) was used for nuclei counterstains.

Studies show that various glial cell types are activated and form the glial scar, in which astrocytes are present in a large proportion after cerebral ischemic injuries in animal models. Astrocytes become hypertrophic, elongate their processes from the penumbra into the infarct core after ischemic stroke, and strongly up-regulate GFAP, a hallmark of astrogliosis responding to the injury. GFAP immunoreactivity in astrocytes is upregulated as early as 1 day after injury and the number of reactive astrocytes is significantly increased around the lesion site at 3-5 days after the injury, but they are absent from the lesion core [6]. Our results indicated that reactive astrocytes were significantly increased in the peri-infarct region, which is consistent with animal studies. Interestingly, no increase in the number of reactive cells in the adjacent normal tissue was found in the human ischemic brain, which is different from the animal models of CNS injury [1]. A recent study showed that human protoplasmic astrocytes were found to be more com- 
plex and significantly larger than the rodent astrocytes [7]. Ablating reactive astrocytes in a mouse model showed a significant increase in lesion size and tissue damage, indicating the beneficial role of reactive gliosis [8, 9]. The glial scars form the borders around the lesion sites and act as protective barriers to infectious agents and inflammatory cells. However, scar formation shows less advantageous outcome in the long term recovery after insults. Reactive gliosis inhibits axonal outgrowth and cellular migration by secreting CSPG and other molecules inhibitory to neurogenesis. Based on the findings in rodents mentioned above, reactive gliosis may play a dual role in brain repair after injuries.

Reactive microglia/macrophages are the second most prominent cell type present within the glial scar, which are seen in the ischemic core within 1 to 2 days after ischemic infarction, and can their presence can extend from the ischemic core into the peri-infarct region over time. Price et al studied the temporal characteristics of microglia activation and found that minimal binding was observed within the first 72 hours after stroke, but tracer binding increased significantly over the first week with some reduction by week 3 to 4 [10]. Microglia cells undergo morphology transformation, releasing pro-inflammatory compounds, and increasingly express immunomodulatory and proliferating markers. Although the role of microglial cells in the development of ischemic insults is not yet clarified, growing evidence suggest that apart from beneficial effects, the pro-inflammatory action of microglia can be detrimental to the injured brain and contribute to infarct evolution.

CSPGs are a complex family of macromolecules that consist of a core protein and one or more covalently attached glycosaminoglycan chains. Reactive gliosis involves a large production of ECM proteins, such as CSPG, which are thus present in areas of reactive gliosis after CNS injury to adult animals. We found that CSPG was increased in the cortical peri-infarct regions in the human brain after ischemic stroke, suggesting that CSPG was also a constituent of the glial scar after ischemic stroke in humans, similar to animal models of CNS injury [11]. Previous studies showed that at the injury site, CSPG was secreted by almost all cell types especially astrocytes [12]. This fact is consistent with our confocal images that showed GFAP-positive cells expressing CSPG, but did not overlap with ED1, indicating that human reactive astrocytes also produced CSPG. CSPGs are known mainly for their growth-inhibitory effects. They are powerful inhibitors of axonal growth cones and neurite extensions [13]. CSPGs-mediated inhibition in a rat model of traumatic spinal cord injury can be overcome with treatment of chondroitinase (Ch)
ABC, which digests glycosaminoglycan chains on CSPGs [14]. Our recent study showed that administration of $\mathrm{ChABC}$, beginning 7 days after focal ischemia, reduces the thickness of the GFAP-immunoreactive glial scar, and improves neurobehavioral outcome in rats [15].

Given the complexity and diversity of human glia, a better understanding about glial cells in the human brain could facilitate future clinical studies and provide novel ways to restore brain functions in patients with various neurological diseases.

\section{Acknowledgements}

This work was partially supported by National Institute of Health (NIH) grants AG21980 and NS057186 (KJ), grants from Zhejiang Provincial Natural Science Foundation of China (R2091137), grants from Zhejiang Provincial Program for the Cultivation of High-level Innovative Health talents (ZBW), and National Natural Science Foundation of China (81171088). Some human tissues were obtained from the NICHD Brain and Tissue Bank for Developmental Disorders at the University of Maryland, Baltimore, MD. The role of the NICHD Brain and Tissue Bank is to distribute tissue and, therefore, cannot endorse the studies performed or the interpretation of results.

\section{Competing Interests}

The authors have declared that no competing interest exists.

\section{References}

1. Nowicka D, Rogozinska K, Aleksy M, Witte OW, Skangiel-Kramska J. Spatiotemporal dynamics of astroglial and microglial responses after photothrombotic stroke in the rat brain. Acta Neurobiol Exp (Wars). 2008; 68: 155-68.

2. Lively S, Moxon-Emre I, Schlichter LC. SC1/hevin and reactive gliosis after transient ischemic stroke in young and aged rats. J Neuropathol Exp Neurol. 2011; 70: 913-29. doi:10.1097/NEN.0b013e318231151e.

3. Morioka T, Kalehua AN, Streit WJ. Characterization of microglial reaction after middle cerebral artery occlusion in rat brain. J Comp Neurol. 1993; 327: 123-32. doi:10.1002/cne.903270110.

4. Rolls A, Shechter R, Schwartz M. The bright side of the glial scar in CNS repair. Nat Rev Neurosci. 2009; 10: 235-41. doi:10.1038/nrn2591.

5. Jin K, Wang X, Xie L, Mao XO, Zhu W, Wang Y, et al. Evidence for stroke-induced neurogenesis in the human brain. Proc Natl Acad Sci U S A. 2006; 103: 13198-202. doi:10.1073/pnas.0603512103.

6. Kawano H, Kimura-Kuroda J, Komuta Y, Yoshioka N, Li HP, Kawamura K, et al. Role of the lesion scar in the response to damage and repair of the central nervous system. Cell Tissue Res. 2012; 349: 169-80. doi:10.1007/s00441-012-1336-5.

7. Oberheim NA, Takano T, Han X, He W, Lin JH, Wang F, et al. Uniquely hominid features of adult human astrocytes. J Neurosci. 2009; 29: 3276-87. doi:10.1523/JNEUROSCI.4707-08.2009.

8. Sofroniew MV. Molecular dissection of reactive astrogliosis and glial scar formation. Trends Neurosci. 2009; 32: 638-47. doi:10.1016/j.tins.2009.08.002

9. Sofroniew MV, Vinters HV. Astrocytes: biology and pathology. Acta Neuropathol. 2010; 119: 7-35. doi:10.1007/s00401-009-0619-8.

10. Price CJ, Wang D, Menon DK, Guadagno JV, Cleij M, Fryer T, et al. Intrinsic activated microglia map to the peri-infarct zone in the subacute phase of $\begin{array}{llll}\text { ischemic } & \text { stroke. } & \text { Stroke. 2006; 37: } & \text { 1749-53. }\end{array}$ doi:10.1161/01.STR.0000226980.95389.0b.

11. Silver J, Miller JH. Regeneration beyond the glial scar. Nat Rev Neurosci. 2004; 5: 146-56. doi:10.1038/nrn1326.

12. McKeon RJ, Jurynec MJ, Buck CR. The chondroitin sulfate proteoglycans neurocan and phosphacan are expressed by reactive astrocytes in the chronic CNS glial scar. J Neurosci. 1999; 19: 10778-88. 
13. Bovolenta P, Fernaud-Espinosa I, Mendez-Otero R, Nieto-Sampedro M. Neurite outgrowth inhibitor of gliotic brain tissue. Mode of action and cellular localization, studied with specific monoclonal antibodies. Eur J Neurosci. 1997; 9: 977-89.

14. Alilain WJ, Horn KP, Hu H, Dick TE, Silver J. Functional regeneration of respiratory pathways after spinal cord injury. Nature. 2011; 475: 196-200. doi:10.1038/nature10199.

15. Hill JJ, Jin K, Mao XO, Xie L, Greenberg DA. Intracerebral chondroitinase ABC and heparan sulfate proteoglycan glypican improve outcome from chronic stroke in rats. Proc Natl Acad Sci U S A. 2012; 109: 9155-60. doi:10.1073/pnas.1205697109. 